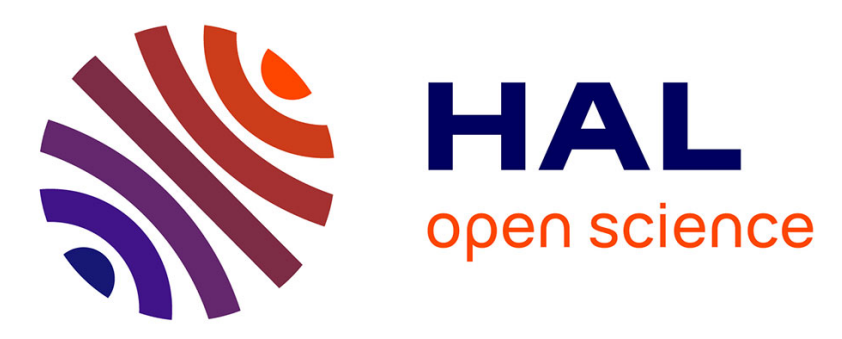

\title{
EXAFS study of rubidium-doped single-wall carbon nanotube bundles
}

Jean-Louis Bantignies, Laurent Alvarez, Raymond Aznar, Robert Almairac, Jean-Louis Sauvajol, L. Duclaux, F. Villain

\section{To cite this version:}

Jean-Louis Bantignies, Laurent Alvarez, Raymond Aznar, Robert Almairac, Jean-Louis Sauvajol, et al.. EXAFS study of rubidium-doped single-wall carbon nanotube bundles. Physical Review B: Condensed Matter and Materials Physics (1998-2015), 2005, 71 (19), pp.195419. 10.1103/PhysRevB.71.195419 . hal-00498755

\section{HAL Id: hal-00498755 \\ https://hal.science/hal-00498755}

Submitted on 13 Dec 2019

HAL is a multi-disciplinary open access archive for the deposit and dissemination of scientific research documents, whether they are published or not. The documents may come from teaching and research institutions in France or abroad, or from public or private research centers.
L'archive ouverte pluridisciplinaire HAL, est destinée au dépôt et à la diffusion de documents scientifiques de niveau recherche, publiés ou non, émanant des établissements d'enseignement et de recherche français ou étrangers, des laboratoires publics ou privés. 


\title{
EXAFS study of rubidium-doped single-wall carbon nanotube bundles
}

\author{
J-L. Bantignies, L. Alvarez, R. Aznar, R. Almairac, and J-L. Sauvajol \\ Groupe de Dynamique des Phases Condensées (UMR CNRS 5581), Université Montpellier II, 34095 Montpellier Cedex 5, France \\ L. Duclaux \\ Centre de Recherche sur la Matière Divisée, CNRS-Université, Orleans, France \\ F. Villain \\ LCIM2, Université Pierre et Marie Curie, 4 Place Jussieu, Paris, France
}

(Received 7 January 2005; published 27 May 2005)

\begin{abstract}
The local structure around the rubidium ions inserted in single-wall carbon nanotube bundles ( $\mathrm{Rb}$-doped SWCNT) is studied by $\mathrm{Rb} K$-edge extended x-ray-absorption fine structure (EXAFS). The dependence of the local order around the rubidium ions is investigated as a function of the time of doping (i.e., as a function of the stoichiometry of the sample). The first coordination shell of the rubidium ions, related to the distance between rubidium and the first nearest-neighboring carbon atoms, has a clear time doping dependence. Comparison between ab initio simulations of the EXAFS spectra and experimental data questions the interstitial site (between three tubes) as the preferential insertion site in SWCNT bundles. The results indicate that the rubidium ions are mainly located inside the tubes and around the bundles. The results are in good agreement with combined $\mathrm{x}$-ray and neutron diffraction experiments performed on the same samples.
\end{abstract}

DOI: 10.1103/PhysRevB.71.195419

PACS number(s): 78.30.Na, 78.70.En

\section{INTRODUCTION}

It has been demonstrated that the physical properties of single-walled carbon nanotube (SWCNT) bundles, such as their conductivity, ${ }^{1}$ can be widely controlled by intercalation by guest species. When the intercalation process is associated with an electronic charge tranfer between the guest species (the dopants) and the nanotube, one says that the SWCNT bundles are doped. Optical absorption investigations ${ }^{2,3}$ and Raman studies ${ }^{4-6}$ performed on alkalimetal doped SWCNT have revealed the electron transfer from the alkali metal to carbon nanotube (generally, the transferred electrons have been considered to fill the $\pi$ band of the nanotube).

Single-wall carbon nanotubes are organized in a 2D triangular lattice of finite size, ${ }^{7,8}$ the so-called SWCNT bundle. The 2D triangular lattice is featured by a (10) Bragg peak, centered around $0.4 \AA^{-1}$ in the x-ray and neutron diffraction diagrams of usual SWCNT samples. ${ }^{7,8}$

Different models of the arrangement of the alkali atoms in doped SWCNT bundles have been proposed. In close-end SWCNTs, the dopants are expected to be inserted into the interstitial channel between the adjacent SWCNTs. In this model, an expansion of the 2D lattice is expected. In agreement with that, some $\mathrm{x}$-ray diffraction (XRD) investigations have shown a Q downshift of the (10) Bragg peak of the 2D lattice in alkali-doped SWCNT bundles. ${ }^{9-11}$ However, recent combined x-ray and neutron diffraction investigations performed on the same sample question the relation between the expansion of the lattice and the position of the (10) Bragg peak in alkali doped SWCNT. ${ }^{12,13}$

Open-end SWCNTs, obtained after an oxydative treatment ${ }^{14-16}$ or by ion irradiation, ${ }^{17}$ may well accept alkali metals to fill their inner cylindrical cavity. Molecular dynamics calculations have predicted that, at low doping level, the alkali metals are inserted between the tubes, and that at high doping level the alkali ions penetrate the inner cavity. ${ }^{18}$ Finally, the sites of the alkali metals around the bundle (in the grooves and at the surface of the tubes) may be also taken into account. Such a site possibility of guest molecules has recently been stated for methane $\left(\mathrm{CH}_{4}\right)$ adsorbed on SWCNT bundles. ${ }^{19}$

The detailed knowledge about the structure of alkalidoped SWCNT, especially the local geometry around the alkali ions, the number of nearest neighbors of alkali ions, and the distances between alkali ions and carbon atoms, are open questions. In the aim to light these points, an extended x-rayabsorption fine structure (EXAFS) investigation has been done. In this paper, we report the EXAFS results obtained on $\mathrm{Rb}$-doped SWCNT (Rb-SWCNT) samples and Rb-doped graphite compounds $\left(\mathrm{RbC}_{8}\right.$ and $\left.\mathrm{RbC}_{24}\right)$. The localization and the ordering of the rubidium ions inside the SWCNT bundle are studied as a function of the doping level. The contribution of the first carbon atom shell surrounding a rubidium ion is used to determine the preferential intercalation sites of $\mathrm{Rb}$ ions within SWCNT bundles.

\section{EXPERIMENT}

\section{A. Samples}

Raw SWCNT samples are prepared by the electric-arc discharge method. ${ }^{20} \mathrm{X}$-ray diffraction experiments are performed to select the flakes of the raw carbon deposit which contain higher amount of tubes assembled into crystalline bundles. In these selected parts, the tube diameters are estimated to be about $1.4 \mathrm{~nm} \pm 0.2 \mathrm{~nm}$. After outgassing and annealing the selected samples at $250{ }^{\circ} \mathrm{C}$ for $24 \mathrm{~h}$ under dynamical vacuum, doping of SWCNT bundles in the vapor phase is achieved in a pyrex reactor by heating rubidium 
metal at $170{ }^{\circ} \mathrm{C}$. A difference of temperature of about $20^{\circ} \mathrm{C}$ is imposed between the alkali-metal reservoir and the SWCNT sample in order to prevent alkali-metal condensation on the sample. Five Rb-doped SWCNT samples are prepared by this way. They differ by the time of doping, and are labelled in the following as: NT2H sample ( $2 \mathrm{~h}$ of doping), NT4H sample (4 h of doping), NT6H sample (6 h of doping), NT60H sample (60 h of doping) and saturation-doped phase (1 week of doping). The $\mathrm{RbC}_{9}$ stoichiometry of the saturation-doped phase is obtained by weight uptake measurements. Two Rb-graphite intercalation model compounds: $\mathrm{RbC}_{8}$ and $\mathrm{RbC}_{24}$, are prepared using the same procedure. For the EXAFS experiments, the samples are transferred in quartz capillaries under argon atmosphere.

\section{B. Experimental set up}

Low-temperature $(10 \mathrm{~K})$ EXAFS experiments are performed at the beamline D42 of the DCI storage ring in the LURE facility. Measurements are performed in transmission mode with a channel cut $\mathrm{Si}(111)$ monochromator. Ionizationchambers were used to detect the signals.

\section{Data analysis}

The EXAFS oscillations, $\chi(k)$, are extracted from x-rayabsorption spectroscopy data using a standard procedure. ${ }^{21,22}$ After the background substraction and edge step normalization of the absorption data, the $\chi(k)$ signals are obtained. The radial structure function $\phi(R)=\mathrm{FT}\left(k^{3} \chi(k)\right)$ is obtained by a Fourier transform of the $k^{3} \chi(k)$. The EXAFS signal in doped-graphite model compounds is strong up to $14 \AA^{-1}$. By contrast, in doped-SWCNT samples the signal to noise ratio is only significant below $10 \AA^{-1}$. In consequence, to compare easily the two sets of data, the Fourier transforms of the $k^{3} \chi(k)$ are performed in the $k$ range from 2.5 to $10 \AA^{-1}$ for all $\mathrm{Rb}$-doped graphite and SWCNT samples.

\section{RESULTS}

In the inset of Fig. 1 are compared the $\mathrm{Rb} K$-edge absorption spectrum, $\mu(E)$, of the saturation phase of $\mathrm{Rb}$-graphite intercalation compound $\left(\mathrm{RbC}_{8}\right)$, and that of the saturation phase of Rb-doped SWCNT $\left(\mathrm{RbC}_{9}\right)$. The EXAFS oscillations are significantly weaker in the Rb-doped SWCNT than that in the Rb-doped graphite. The EXAFS oscillations of the $\mathrm{Rb}$-doped graphite are strong and well-defined in agreement with the high degree of crystallinity of this intercalated graphite compound. ${ }^{23}$ By contrast, the weak EXAFS oscillations in the Rb-doped SWCNT sign the presence of a strong local disorder around the rubidium ions. Figure 1 displays the EXAFS oscillations, $\chi(k)$, measured on the different Rbdoped SWCNT samples (top), and on the two Rb-graphite intercalation compounds, $\mathrm{RbC}_{24}$ and $\mathrm{RbC}_{8}$ (bottom).

First, we are interested in the EXAFS results carried out on the $\mathrm{Rb}$-graphite intercalation model compounds. The $\mathrm{RbC}_{8}$ EXAFS signal is stronger than the $\mathrm{RbC}_{24}$ EXAFS signal. An important result is that the EXAFS oscillations $\chi(k)$ measured on $\mathrm{RbC}_{24}$ and $\mathrm{RbC}_{8}$ are significantly different (Fig.
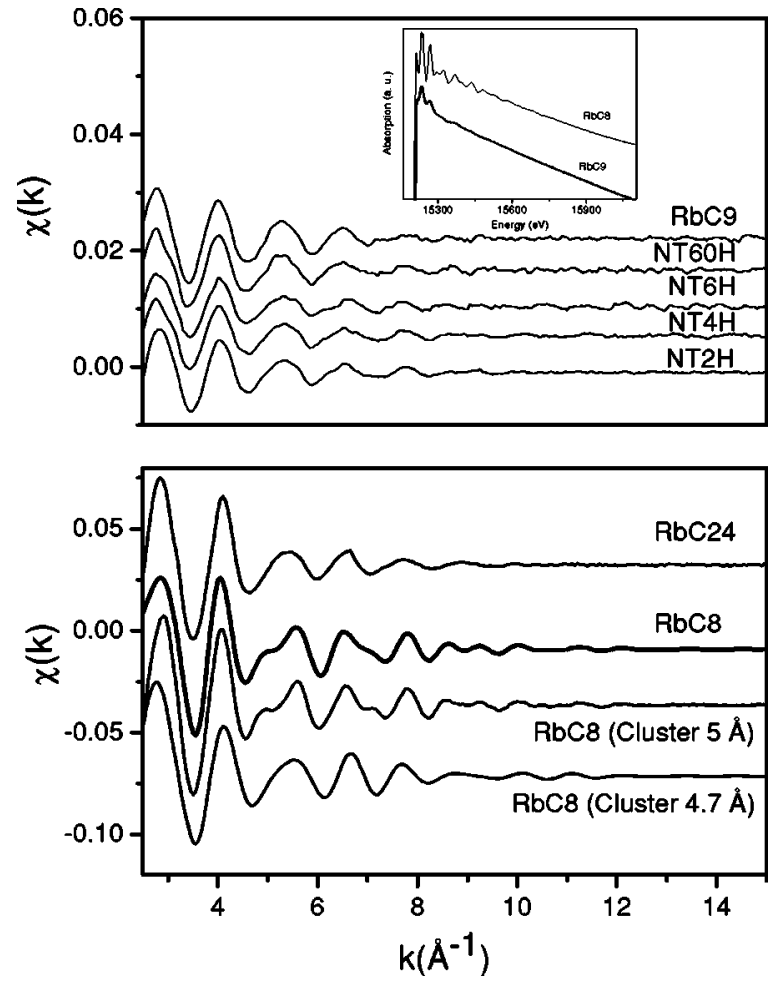

FIG. 1. The EXAFS oscillations $\chi(k)$ of Rb-doped SWCNT samples (top). The EXAFS oscillations $\chi(k)$ of $\mathrm{RbC}_{24}$ and $\mathrm{RbC}_{8}$ intercalation graphite compounds (bottom). Comparison between experimental results and FEFF8 calculations is done. The first simulation is performed with a cluster of 4 shells up to $5 \AA$ including the $\mathrm{Rb}-\mathrm{Rb}$ contributions. The second simulation is performed by considering the three carbon shells up to $4.7 \AA$ from the adsorbing atom. Inset: $\mathrm{Rb} K$-edge absorption spectra of $\mathrm{RbC}_{8}$ and $\mathrm{RbC}_{9}$. Spectra are vertically shifted for clarity.

1 , bottom). The differences of the $\chi(k)$ profiles can be understood in terms of differences in the structural organizations of the rubidium and graphite lattices. In $\mathrm{RbC}_{24}$, at $T$ $<156 \mathrm{~K}$, the alkali metal lattice can be viewed as an incommensurate modulated structure with respect to the graphite matrix. ${ }^{24,25}$ By contrast, in $\mathrm{RbC}_{8}$, the rubidium and graphite lattices are commensurate. ${ }^{26,27}$ In consequence, a distribution of the $\mathrm{Rb}-\mathrm{C}$ distances is expected in $\mathrm{RbC}_{24}$.

EXAFS oscillations in $\mathrm{Rb}$-graphite intercalation model compounds have been calculated as a function of the diameter of the cluster by using $a b$ initio FEFF8 simulations. ${ }^{28}$

A general good agreement between experimental and simulation data was obtained in the framework of a single scattering process, and any improvement was obtained by taking into account multiple scattering processes. First, one can qualitatively reproduce the main EXAFS features of $\mathrm{RbC}_{8}$ by considering a cluster diameter of $10 \AA$ (Fig. 1, bottom). A good agreement between simulated and experimental spectra is found by taking into account the three first carbon coordination shells around a rubidium ion, centered at $3.16 \AA$ (with 12 carbon atoms), $4.00 \AA$ (with 12 carbon atoms), and $4.70 \AA$ (with 24 carbon atoms), respectively. Furthermore, the first $\mathrm{Rb}-\mathrm{Rb}$ coordination shell formed by six rubidium ions centered at $4.92 \AA$ has to be included in the cluster to simulate accurately the EXAFS signal in $\mathrm{RbC}_{8}$. 

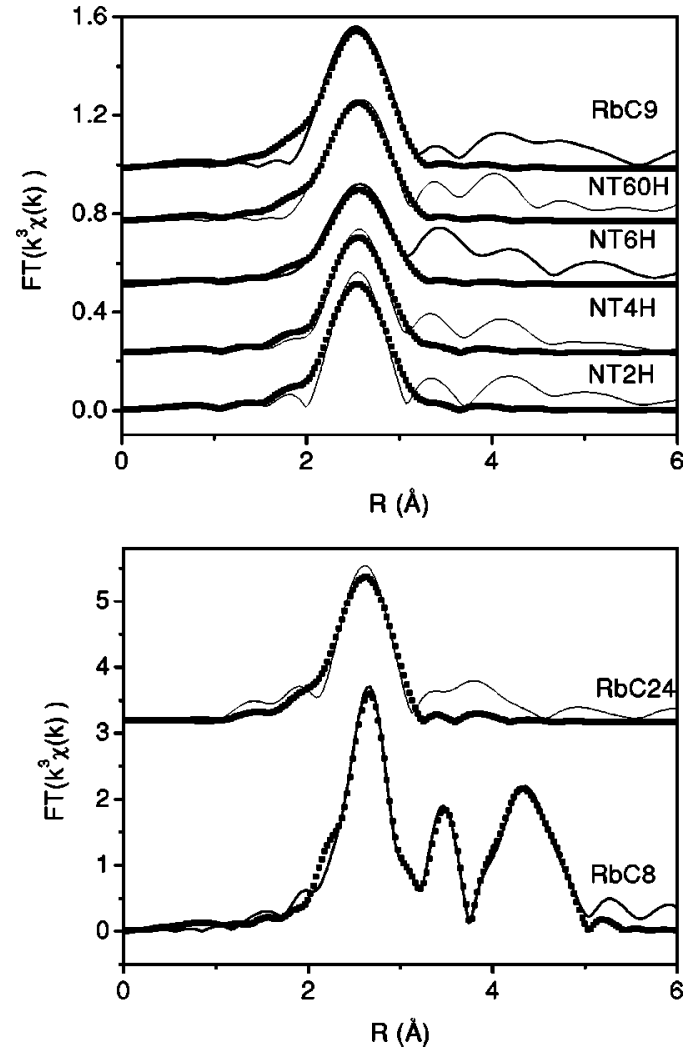

FIG. 2. The pseudoradial functions, $\mathrm{FT}\left(\mathrm{k}^{3} \chi(k)\right)$ of the different Rb-doped SWCNT (top) and Rb-doped graphite compounds: $\mathrm{RbC}_{24}$ and $\mathrm{RbC}_{8}$ (bottom). The fits are performed using backscattering amplitude and total phase shift extracted from FEFF8 calculations (square). Note that the difference between the peak position $(2.7 \AA)$ and the real $\mathrm{Rb}-\mathrm{C}$ distance $(3.15 \AA)$ is due to a phase term.

The simulation considering only the three first carbon shells is close to the experimental spectrum of $\mathrm{RbC}_{24}$. This indicates that the $\mathrm{Rb}-\mathrm{Rb}$ contributions are not detected in $\mathrm{RbC}_{24}$. This latter result is assigned to the signature of the disorder of the rubidium ions in $\mathrm{RbC}_{24}$. All these results are further corroborated by the analysis of the pseudoradial distributions, $\mathrm{FT}\left(k^{3} \chi(k)\right)$, derived from the Fourier transform of the EXAFS spectra (Fig. 2, bottom). The main peak at the lowest $R$ value corresponds to the shortest $\mathrm{Rb}-\mathrm{C}$ distance. In $\mathrm{RbC}_{8}$, the second peak is assigned to the shell of the second nearest-neighbor carbon atoms, and the third peak combines contributions from the third-nearest neighbor carbon atoms, and $\mathrm{Rb}-\mathrm{Rb}$ distances.

Standard theoretical amplitudes and phase functions are extracted from FEFF8 simulations. For each shell $j$, the fit gives information on the coordination number, $N_{j}$, the bond distances $R_{j}$, the Debye-Waller factor $\sigma_{j}^{2}$, and the energy shift $\Delta E_{0}^{j}$. The strong correlation between $N_{j}$ and $\sigma_{j}^{2}$ is a well known problem of the analysis of EXAFS data. ${ }^{29}$ The reliability of the averaged $N_{j}$ values was checked by fitting the low temperature data for different $k$ spaces. Since $N_{j}$ and $\sigma_{j}^{2}$ depend on $k$ differently, it is expected that the fit with a properly fixed value of $N_{j}$ leads to a same value of $\sigma_{j}^{2}$ for different $k$-spaces. For $\mathrm{RbC}_{8}$, the fit procedure yields to structural parameters in agreement with the crystallographic parameters. The Debye-Waller $\sigma_{j}^{2}$ values are $0.006 \AA^{2}$ for the
TABLE I. Structural parameters deduced from the least squares fit of the first shell of the Rb-doped graphite samples. $N$ is the coordination number, $R$ the fitted $\mathrm{Rb}-\mathrm{C}$ distance, $\sigma^{2}$ the DebyeWaller factor, $\Delta E$ the energy shift.

\begin{tabular}{ccc}
\hline \hline & $\mathrm{RbC} 8$ & $\mathrm{RbC} 24$ \\
\hline$N$ & $12 .(1)$ & $12 .(1)$ \\
$\sigma^{2}$ & 0.006 & 0.009 \\
$\Delta E$ & -1.45 & -2.89 \\
$R$ & $3.15(1)$ & $3.15(1)$ \\
\hline \hline
\end{tabular}

two shorter $\mathrm{Rb}-\mathrm{C}$ distances and 0.008 and $0.010 \AA^{2}$ for the largest $\mathrm{Rb}-\mathrm{C}$, and $\mathrm{Rb}-\mathrm{Rb}$ distances. For $\mathrm{RbC}_{24}$, the fit of the data by considering the contributions from the three first carbon coordination shells leads to unphysical values of the different parameters. In $\mathrm{RbC}_{24}$, the significant EXAFS signal only originates in the contribution of the first carbon coordination shell. In consequence, in the following, only the position, width and intensity of the first peak of $\operatorname{FT}\left(k^{3} \chi(k)\right)$ will be discussed. For the first coordination shell, a comparison of the results of the fit for $\mathrm{RbC}_{24}$ and $\mathrm{RbC}_{8}$, is reported in Table I. A large Debye-Waller factor is obtained in $\mathrm{RbC}_{24}$. This value reflects the distribution of the $\mathrm{Rb}-\mathrm{C}$ distances in the modulated structure of the alkali ions in $\mathrm{RbC}_{24}$ compound. This distribution prevents the analysis of the structural order at longer distances. Both the first $\mathrm{Rb}-\mathrm{C}$ distance in $\mathrm{RbC}_{24}$ graphite intercalation compound (similar to the one in $\mathrm{RbC}_{8}$ ), and the number of carbon neighbors indicate that the $\mathrm{Rb}$ atoms lie preferentially in the center of hexagons. This result is in agreement with the model of Clarke et al. (commensurate domains separated by discommensuration walls), ${ }^{30}$ or the Dicenzo's one (relaxed incommensurate structure). ${ }^{31}$ In the following, on the basis of these latter results and with regards to the intrinsic disorder of the $\mathrm{Rb}$ doped SWCNT compounds, we restrict our analysis of the EXAFS data obtained on Rb-doped SWCNT to the first carbon coordination shell of the rubidium ion.

First, it must be pointed out that the EXAFS oscillations, $\chi(k)$, measured on the five Rb-doped SWCNT samples are close to each other. As expected, one only observes an increase of the signal to noise ratio with the doping level. All the $\chi(k)$ profiles mainly exhibit a single oscillation assigned to a single coordination shell. To determine the structural parameters of the Rb-doped SWCNT compounds, we use the same procedure that the one previously reported to fit the EXAFS data on $\mathrm{Rb}$-doped graphite compounds. The results are reported in Table II for the first coordination shell. In all the samples, the average $\mathrm{Rb}-\mathrm{C}$ distance (called $R$ in the tables) is found around $3.15 \AA$, in good agreement with the one derived from $x$-ray diffraction data obtained on the same samples. ${ }^{12}$ More striking, the number of first nearest neighbors, $N$, is clearly dependent of the doping level. This number increases with the doping level (related to the time of doping).

In saturation phase, the fit shows that rubidium ions are surrounded by six carbon atoms, with an average $\mathrm{Rb}-\mathrm{C}$ distance of about $3.16 \AA$. The value of this distance, and the coordination number, suggest that a rubidium ion is facing a 
TABLE II. Structural parameters deduced from the least squares fit of the first shell of the Rb-doped SWCNT samples.

\begin{tabular}{cccccc}
\hline \hline \multicolumn{2}{c}{ NTH2 } & NTH4 & NTH6 & NTH60 & RbC9 \\
\hline$N$ & $2.8(5)$ & $2.4(5)$ & $2.8(5)$ & $4.0(5)$ & $5.5(5)$ \\
$\sigma^{2}$ & 0.008 & 0.007 & 0.011 & 0.013 & 0.015 \\
$\Delta E$ & -5.40 & -5.33 & -3.55 & -4.57 & -5.02 \\
$R$ & $3.15(1)$ & $3.15(1)$ & $3.14(2)$ & $3.16(1)$ & $3.16(1)$ \\
\hline \hline
\end{tabular}

carbon hexagon. Then, in this sample, the local order of rubidium ions is close to the one in the saturation phase of the Rb-doped graphite compounds. For low, and intermediate doping levels, the preferential site is not clearly identified. However, the number of coordination is significantly lower than 6 (Table II). Consequently, one assumes that the rubidium ions are located off center of the carbon hexagons.

In all the samples, the Debye-Waller factor, $\sigma_{j}$, is large, and it increases with the doping level. It goes from $0.008 \AA^{2}$ in $\mathrm{NT} 2 \mathrm{H}$ to $0.015 \AA^{2}$ in $\mathrm{RbC}_{9}$. With regard to the small
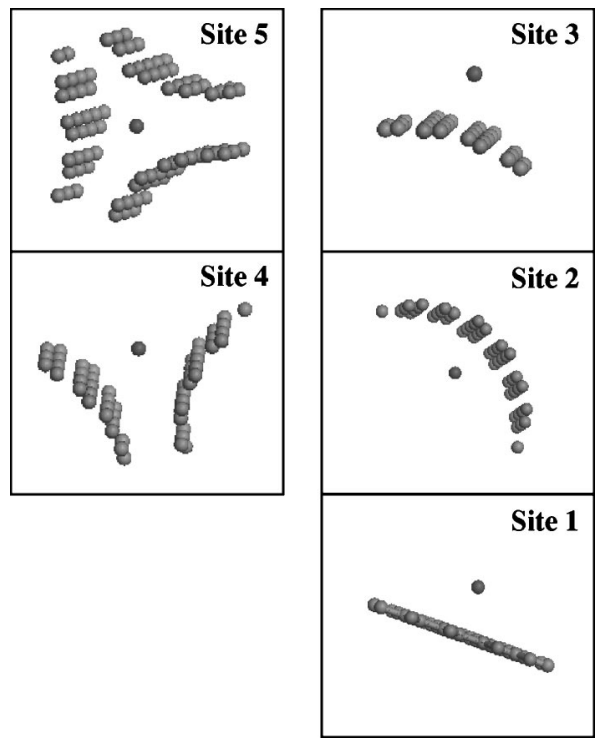

FIG. 3. The different local structures used in the FEFF calculations for clusters of $4.7 \AA$ around $\mathrm{Rb}$. The curvature corresponds to the one of a $(10,10)$ nanotube. For site 1 ( $R b$ on graphene sheet), the first coordination shell is related to a $\mathrm{Rb}-\mathrm{C}$ distances of $3.16 \AA$, and the number of carbons on this shell is six. The second coordination shell is related to a $\mathrm{Rb}-\mathrm{C}$ distance at $4 \AA$, with six carbon atoms on this shell. The third $\mathrm{Rb}-\mathrm{C}$ distance coordination shell is related to a $\mathrm{Rb}-\mathrm{C}$ distance of $4.70 \AA$, with twelve carbon atoms on this shell. For site 2 ( $R b$ on inner SWCNT surface), six $\mathrm{Rb}-\mathrm{C}$ distances are found in the 3.016-3.16 $\mathrm{A}$ range, six $\mathrm{Rb}-\mathrm{C}$ distances between 3.5-4 $\mathrm{A}$, and twelve $\mathrm{Rb}-\mathrm{C}$ distances between 4 and $4.7 \AA$. For site 3 ( $\mathrm{Rb}$ on outer SWCNT surface) six $\mathrm{Rb}-\mathrm{C}$ distances are found in the 3.12-3.23 $\AA$ range, eight $\mathrm{Rb}-\mathrm{C}$ distances between 4 and $4.7 \AA$. For site 4 ( $\mathrm{Rb}$ on groove) four $\mathrm{Rb}-\mathrm{C}$ distances are found between 3.11-3.28 $\mathrm{A}$, six $\mathrm{Rb}-\mathrm{C}$ distances from 3.5 to $4.7 \AA$, and eighteen from 3.9 to $4.7 \AA$. For site 5 (Rb in interstitial site), ten $\mathrm{Rb}-\mathrm{C}$ distances are found between $2.77-3.31 \AA$, sixteen $\mathrm{Rb}-\mathrm{C}$ distances from 3.6 to $3.98 \AA$, and fifteen from 4.15 to $4.7 \AA$. displacement amplitudes of the atoms at low-temperature, the Debye-Waller factor, $\sigma_{j}^{2}$, is expected to be weak at $10 \mathrm{~K}$. In consequence, the large value of the Debye-Waller factor is assigned to the contribution of the static disorder of the carbon atoms around a rubidium ion. The origin of the distribution of the $\mathrm{Rb}-\mathrm{C}$ distances could be the distorsion of the carbon hexagon due to the insertion of rubidium. Such a deformation was reported under the insertion of endohedral ions located in front of hexagon inside fullerene cages. ${ }^{32}$.

To guide our understanding of the experimental data, we have performed $a b$ initio FEFF8 simulations of the EXAFS oscillations, $\chi(k)$ for different possible insertion sites of the rubidium ions in Rb-doped graphite and SWCNT compounds (Fig. 3). Because the EXAFS signal is not sensitive to $\mathrm{Rb}-\mathrm{C}$ distances greater than $4.70 \AA$ in the majority of the present compounds, the EXAFS oscillations are simulated by considering a diameter of clusters of $4.70 \AA$. In all the calculations, we impose the first $\mathrm{Rb}-\mathrm{C}$ distance to be close to the one derived from the previous EXAFS analysis of the first coordination shell.

First, we test the curvature effect on $\chi(k)$ by calculating the EXAFS oscillations for $\mathrm{Rb}$ ion in front of a flat graphite layer (Fig. 3, Site 1), and in front of a curved graphite layer (Fig. 3, Sites 2 and 3). As displayed in Fig. 4, simulations clearly bring out the dependence of $\chi(k)$ with the curvature of the graphite layer. We have also simulated the EXAFS spectra for a rubidium ion located in a groove site around the bundle (Fig. 3, Site 4), and in the interstitial space (between 3 tubes) inside the bundle (Fig. 3, Site 5). The sensitivity of the EXAFS oscillations to the different insertion sites of a

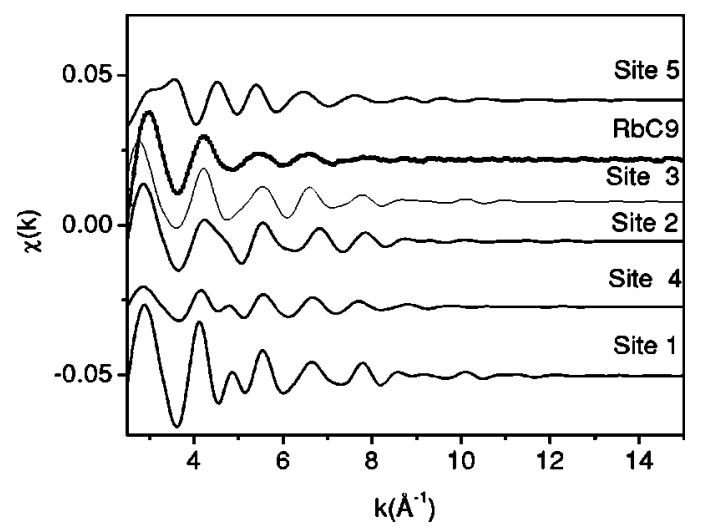

FIG. 4. Comparison between the experimental Rb $K$-edge EXAFS oscillations, $\chi(k)$, measured in the saturation-phase of Rbdoped SWCNT $\left(\mathrm{RbC}_{9}\right)$, and FEFF8 calculations for the different $\mathrm{Rb}$ sites. 
rubidium ion is displayed in Fig. 4. The comparison of simulations with experimental data carried out on the saturationphase of Rb-doped SWCNT $\left(\mathrm{RbC}_{9}\right)$ allows to conclude that the interstitial voids are not the preferential sites of the rubidium ions in $\mathrm{RbC}_{9}$ (compare the top curve to the experimental one in Fig. 4). By contrast, taking into account the position of the rubidium ions at the inner surface of the tubes, and around the bundle, significantly improves the simulation-experience agreement.

\section{CONCLUSION}

The interstitial site, which is usually reported as the preferential site of insertion of alkali atoms in alkali-doped SWCNT bundle ${ }^{11}$ seems to be ruled out from the EXAFS results. The first argument to rule out this site is that the number of carbon at a $\mathrm{Rb}-\mathrm{C}$ distance below $4 \AA$ is found to be six. This number is significantly smaller than the one expected [around 26 for a $(10,10)$ bundles] for rubidium ion localized in the interstitial space. Another significant argument is the complete disagreement between the simulation result (Fig. 4, Site 5) and the experimental EXAFS oscillations, $\chi(k)$ (Fig. 4, thick solid line). On the other hand, the non expansion of the 2D triangular lattice under the intercalation of rubidium in SWCNT bundles, recently stated from neutron diffraction experiment, ${ }^{12}$ prevents a position of rubidium ions in between two tubes. In this latter assumption, the expected number of coordination would be greater than 6 , in opposition with the EXAFS results. The comparison between simulation and experiment suggests that the rubidium ions can be located around the bundles (grooves and surface). However, with regard to the overall composition at saturation determined experimentally, it is unlikely that rubidium ions are only located in this kind of sites. It is generally assumed that the alkali ions cannot be located in the inner cavity of close-end tubes. However, the simulation of EXAFS data do not exclude this site (Fig. 4, site 2). Although we do not have other experimental evidences about the filling of the tube in the sample under consideration, we suggest that rubidium ions are also inserted inside the inner cavity of the tubes. It can be pointed out that the question of the stoichiometry of the sample is solved if one considers rubidium ions located around the bundles and inside the tubes.

\section{ACKNOWLEDGMENTS}

This work was achieved under the proposals of LURE (Orsay, France). The authors thank S. Rols and T. Michel for his help in the building of the clusters used in the simulations.
${ }^{1}$ R. S. Lee, H. J. Kim, A. Thess, and R. E. Smalley, Nature (London) 388, 255 (1997).

${ }^{2}$ P. Petit, C. Mathis, C. Journet, and P. Bernier, Chem. Phys. Lett. 305, 370 (1999).

${ }^{3}$ S. Kazaoui, N. Minami, R. Jacquemin, H. Kataura, and Y. Achiba, Phys. Rev. B 60, 13339 (1999).

${ }^{4}$ A. M. Rao, P. C. Eklund, S. Bandow, A. Thess, and R. E. Smalley, Nature (London) 388, 257 (1997).

${ }^{5}$ N. Bendiab, L. Spina, A. Zahab, P. Poncharal, C. Marlière, J.-L. Bantignies, E. Anglaret, and J.-L. Sauvajol, Phys. Rev. B 63, 153407 (2001).

${ }^{6}$ N. Bendiab, E. Anglaret, J.-L. Bantignies, A. Zahab, J.-L. Sauvajol, P. Petit, C. Mathis, and S. Lefrant, Phys. Rev. B 64, 245424 (2001).

${ }^{7}$ A. Thess, R. Lee, P. Nikolaev, H. J. Dai, P. Petit, J. Robert, C. H. Xu, Y. H. Lee, S. G. Kim, A. G. Rinzler, D. T. Colbert, G. E. Scuseria, D. Tomanek, J. E. Fischer, and R. E. Smalley, Science 273, 483 (1996).

${ }^{8}$ S. Rols, R. Almairac, L. Henrard, E. Anglaret, and J.-L. Sauvajol, Eur. Phys. J. B 10, 263 (1999).

${ }^{9}$ T. Pichler, M. Sing, M. Knupfer, M. S. Golden, and J. Fink, Solid State Commun. 109, 721 (1999).

${ }^{10}$ C. Bower, A. Kleinhammes, Y. Wu, and O. Zhou, Chem. Phys. Lett. 288, 481 (1998).

${ }^{11}$ L. Duclaux, Carbon 40, 1751 (2002), and references therein.

${ }^{12}$ N. Bendiab, Ph.D. thesis, University of Montpellier, 2003.

${ }^{13}$ S. Challet, P. Azais, R. J-M. Pellenq, and L. Duclaux, Chem. Phys. Lett. 377, 544 (2003)

${ }^{14}$ X. Fan, E. C. Dickey, P. Eklund, K. Williams, L. Grigorian, R.
Buczko, S. T. Pantelides, and S. J. Pennycook, Phys. Rev. Lett. 84, 4621 (2000).

${ }^{15}$ H. Shimoda, B. Gao, X. P. Tang, A. Kleinhammes, L. Fleming, Y. Wu, and O. Zhou, Phys. Rev. Lett. 88, 015502 (2002).

${ }^{16}$ L. Duclaux, J.-P. Salvetat, P. Lauginie, T. Cacciaguera, A. M. Faugère, C. Goze-Bac, and P. Bernier, J. Phys. Chem. Solids 64, 571 (2003).

${ }^{17}$ G. H. Jeong, R. Hatakeyama, T. Hirata, K. Tohji, K. Motomiya, T. Yaguchi, and Y. Kawazoe, Chem. Commun. (Cambridge) 1, 152 (2003).

${ }^{18}$ G. Gao, T. Cajin, and William A. Goddard III, Phys. Rev. Lett. 80, 5556 (1998).

${ }^{19}$ M. R. Johnson, S. Rols, P. Wass, M. Muris, M. Bienfait, P. Zeppenfeld, and N. Dupont-Pavlovsky, Chem. Phys. 293, 217 (2003).

${ }^{20}$ C. Journet, W. Maser, P. Bernier, A. Loiseau, M. L. de la Chapelle, S. Lefrant, P. Deniard, R. Lee, and J. E. Fischer, Nature (London) 388, 756 (1997).

${ }^{21}$ P. A. Lee, P. H. Citrin, P. Eisenberger, and B. M. Kincaid, Rev. Mod. Phys. 53, 769 (1981).

${ }^{22}$ B. Lengeler and P. Eisenberger, Phys. Rev. B 21, 4507 (1980).

${ }^{23}$ J. Bouat, D. Bonnin, L. Facchini, and F. Beguin, Synth. Met. 7, 233 (1983).

${ }^{24}$ M. Dresselhaus and G. Dresselhaus, Adv. Phys. 30, 139 (1981).

${ }^{25}$ F. Rousseaux, R. Moret, D. Guerard, and P. Lagrange, Phys. Rev. B 42, 725 (1990).

${ }^{26}$ W. Rüdorff, and E. Schulze, Z. Anorg. Allg. Chem. 277, 156 (1954).

${ }^{27}$ D. E. Nixon and G. S. Parry, J. Phys. D 1, 291 (1968). 
${ }^{28}$ A. L. Ankudinov, B. Ravel, J. J. Rehr, and S. D. Conradson, Phys. Rev. B 58, 7565 (1998).

${ }^{29}$ Boon K. Tio, EXAFS: Basic Principles and Data Analysis (Springer Verlag, Berlin, 1986).

${ }^{30}$ R. Clarke, N. Caswell, and S. A. Solin, Phys. Rev. Lett. 42, 61
(1979).

${ }^{31}$ S. B. DiCenzo, Phys. Rev. B 26, 5878 (1982).

${ }^{32}$ H. Giefers, F. Nessel, S. I. Gyory, M. Strecker, G. Wortmann, Yu. S. Grushko, E. G. Alekseev, and V. S. Kozlov, Carbon 37, 721 (1999). 